\title{
Far-detuned cascaded intermodal four-wave mixing in a multimode fiber
}

\author{
R. Dupiol, ${ }^{1,2, \dagger}$ A. Bendahmane, ${ }^{1, \dagger}$ K. Krupa, ${ }^{1,2}$ A. Tonello, ${ }^{2}$ M. Fabert, ${ }^{2}$ B. Kibler, ${ }^{1}$ T. Sylvestre, ${ }^{3}$ \\ A. Barthelemy, ${ }^{2}$ V. Couderc, ${ }^{2}$ S. Wabnitz, ${ }^{4,5}$ and G. Millot ${ }^{1, *}$ \\ ${ }^{1}$ Université Bourgogne Franche-Comté, ICB UMR CNRS 6303, 9 Avenue A. Savary, 21078 Dijon, France \\ 2Université de Limoges, XLIM, UMR CNRS 7252, 123 Avenue A. Thomas, 87060 Limoges, France \\ ${ }^{3}$ Université Bourgogne Franche-Comté, Institut FEMTO-ST, UMR CNRS 6174, 15B Avenue des Montboucons, 25030 Besançon, France \\ ${ }^{4}$ Università di Brescia, Dipartimento di Ingegneria dell'Informazione and INO-CNR, via Branze 38, 25123 Brescia, Italy \\ ${ }^{5}$ Novosibirsk State University, 1 Pirogova str., Novosibirsk 630090, Russia \\ ${ }^{*}$ Corresponding author: Guy. Millot@u-bourgogne.fr
}

Received 20 January 2017; revised 28 February 2017; accepted 1 March 2017; posted 3 March 2017 (Doc. ID 285276); published 23 March 2017

We demonstrate far-detuned parametric frequency conversion processes in a few mode graded-index optical fibers pumped by a $Q$-switched picosecond laser at $1064 \mathrm{~nm}$. Through a detailed analytical and numerical analysis, we show that the multiple sidebands are generated through a complex cascaded process involving inter-modal four-wave mixing. The resulting parametric wavelength detuning spans in the visible down to $405 \mathrm{~nm}$ and in the nearinfrared up to $1355 \mathbf{n m}$. ( 2017 Optical Society of America

OCIS codes: (190.4370) Nonlinear optics, fibers; (190.4410) Nonlinear optics, parametric processes; (190.4380) Nonlinear optics, four-wave mixing.

https://doi.org/10.1364/OL.42.001293

Multimode fibers (MMFs) recently have attracted renewed interest in the context of spatial-division multiplexing (SDM) for increasing the capacity of fiber-based communications [1]. Research on MMF led to new nonlinear spatiotemporal phenomena such as multimode solitons, geometric parametric instability (GPI), supercontinuum (SC) generation, and selfinduced beam cleaning [2-12]. Among MMFs, parabolic graded-index multimode fibers (GRIN-MMFs) have the unique property of supporting guided modes with equally spaced propagation constants and nearly identical group velocities $[3,4]$. These features enabled the observation of multimode solitons [5] and the associated generation of a set of discrete dispersive waves over an ultra-broadband range [6-8].

In this context, much work has focused on the dynamics of inter-modal four-wave mixing (IMFWM) in MMFs, which allows for large frequency shifts (hundreds of $\mathrm{THz}$ ) when compared with single-mode fibers (SMFs) $[4,9,10]$. When a large number of modes is initially excited in MMFs, the frequencies of the parametric lines can be determined through a collective approach involving the dynamics of the total field, as proposed by Longhi [11]. The longitudinal spatial intensity oscillations of a multimode beam give rise to GPI and multiple sidebands that can be generated far away from the pump (shifts larger than $100 \mathrm{THz}$ ) [11,12]. This self-induced phase-matching mechanism is analogous to quasi-phase matched wave (QPM) generation in periodic amplified transmission systems or dispersion-managed fibers [13-15]. For a few-mode fiber (FMF) instead, the number of intermodal, nonlinear coupling terms is much smaller: the frequencies of parametrically generated waves can be obtained from the phase-matching conditions of IMFWM processes. Mafi and co-workers recently revisited the theory of IMFWM in a GRIN-FMF for a degenerate spatial mode pump configuration, in which two pump photons are in the same spatial mode [3,16,17], and derived a new analytic formula for the parametric sideband frequencies, their modal composition, and associated conversion efficiency.

In this Letter, we experimentally investigate IMFWM in a GRIN-FMF pumped in the normal dispersion regime at $1064 \mathrm{~nm}(282 \mathrm{THz})$. We report the observation of parametric sidebands ranging from the visible down to $405 \mathrm{~nm}$ to the nearinfrared up to $1355 \mathrm{~nm}$, which is the largest frequency range observed to date in a FMF. The analytical phase-matching condition for IMFWM allows us to predict the position of the first observed spectral component, which is largely detuned from the pump by $198 \mathrm{THz}$. Next, such a powerful first anti-Stokes sideband acts as a secondary pump. We can accurately predict most of the remaining observed parametric sidebands based on the hypothesis that they are generated by a cascaded IMFWM process. Our results are well supported by numerical simulations based on the integration of a multidimensional nonlinear Schrödinger equation.

To experimentally investigate IMFWM in MMFs, we used a 1-m-long piece of GRIN-FMF [18]. This fiber was specially designed to support two groups of modes $\left(\mathrm{LP}_{01}\right.$ and $\left.\mathrm{LP}_{11}\right)$ at $1550 \mathrm{~nm}$, with linear losses below $0.22 \mathrm{~dB} / \mathrm{km}$. Figure 1 (a) shows the refractive index profile of the fiber measured at $632 \mathrm{~nm}$. It exhibits a parabolic central shape that can be well fitted [red line in Fig. 1(a)] by the analytic formula: $n^{2}(\rho)=n_{o}^{2}$ $\left[1-2 \Delta(\rho / R)^{2}\right]$, with $n_{o}=1.457, R=11 \mu \mathrm{m}$, and $\Delta=$ 0.0089 . Even though the fiber is nominally bimodal at 


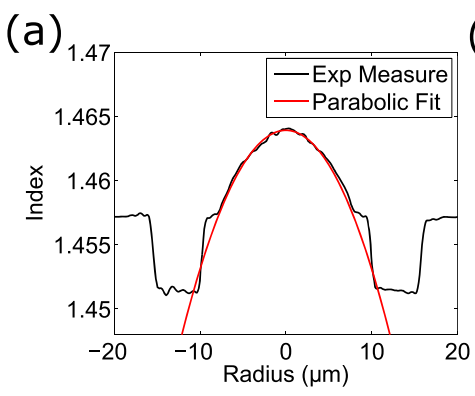

(b)

(c)

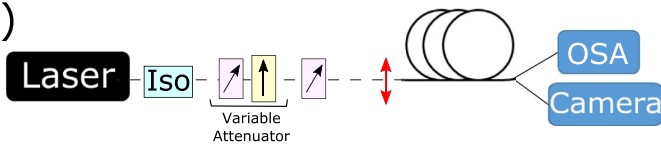

Fig. 1. (a) Refractive index profile measured at $632 \mathrm{~nm}$. The red line indicates a parabolic fit of the central part (see text). (b) Spatial group of modes experimentally identified at $1064 \mathrm{~nm}$. (c) Experimental setup. ISO, isolator; OSA, optical spectrum analyzer.

$1550 \mathrm{~nm}$, we experimentally identified two additional groups of modes $\left(\mathrm{LP}_{02}\right.$ and $\left.\mathrm{LP}_{21}\right)$ at $1064 \mathrm{~nm}$, as shown in Fig. 1(b). Consequently, the GRIN fiber can still be considered as a FMF at this wavelength where it exhibits strong normal dispersion. Figure 1(c) shows a scheme of our experimental setup. For a pump laser, we used a $Q$-switched microchip Nd:YAG laser delivering $400 \mathrm{ps}$ pulses at $1064 \mathrm{~nm}$ with a repetition rate of $1 \mathrm{kHz}$. The maximum peak power of the emitted pulses is $220 \mathrm{~kW}$. The polarization state and power of the pulses are controlled by the combination of a polarizer and two half-wave plates. Light is then coupled into the fiber through a $10 \times$ microscope objective. At the fiber output, the optical spectrum is recorded by use of three Yokogawa optical spectrum analyzers (OSA) covering a wide range from 350 to $2400 \mathrm{~nm}$. A CMOS camera is also used to spatially characterize the output beam intensity profiles.

We first performed a set of measurements by increasing the pump power. Careful attention was paid to the injection conditions, to limit the number of involved modes in the FWM process and to ensure that the pump beam was mainly guided in the $\mathrm{LP}_{01}$ mode. Figure 2(a) shows four experimental spectra obtained at the fiber output when increasing the peak power from $44 \mathrm{~kW}$ to $80 \mathrm{~kW}$. For a peak power of $44 \mathrm{~kW}$ (blue spectrum), we observed the generation of four sidebands around the pump. Two of them (1038 and $1090 \mathrm{~nm}$ ) come from intermodal modulational instability involving the pump in a nondegenerate mode [19,20], while those at 1016 and $1116 \mathrm{~nm}$, detuned by $\pm 13.2 \mathrm{THz}$, are their second orders seeded by stimulated Raman scattering (SRS). More remarkably, we also observed a narrowband far-detuned sideband into the visible at $625.2 \mathrm{~nm}$, up-shifted by $198 \mathrm{THz}$ from the pump [blue line, Fig. 2(a)], owing to intermodal phase matching with a degenerate pump configuration. When increasing the peak power, the conversion efficiency into this visible sideband at $625.2 \mathrm{~nm}$ increases substantially. For a peak power of $50 \mathrm{~kW}$ [green spectrum in Fig. 2(a)], the visible sideband begins to saturate, while its bandwidth progressively broadens. We further observed new far-detuned spectral peaks at $555.2 \mathrm{~nm}(268.4 \mathrm{THz})$ and $512.6 \mathrm{~nm}(303.3 \mathrm{THz})$, and other peaks at $800.1 \mathrm{~nm}(93 \mathrm{THz})$ and $1355.1 \mathrm{~nm}(-60.5 \mathrm{THz})$. When we further raised the pump power above $59 \mathrm{~kW}$, we suddenly observed six additional
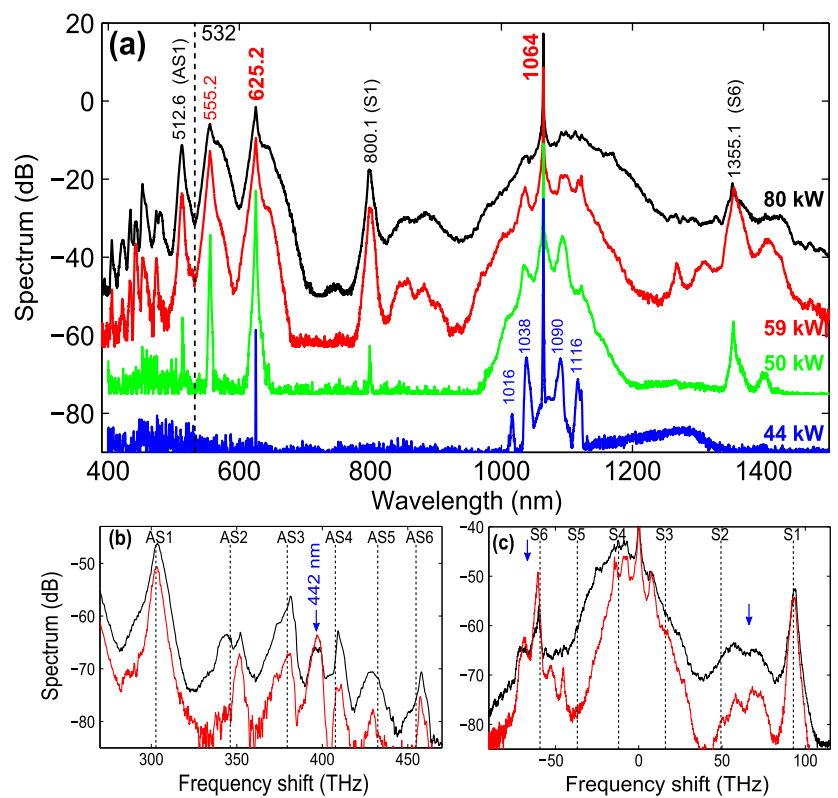

Fig. 2. (a) Output spectra recorded for a pump peak power of $44 \mathrm{~kW}$ (blue), $50 \mathrm{~kW}$ (green), $59 \mathrm{~kW}$ (red), and $80 \mathrm{~kW}$ (black). (b, c) Zoom on (b) the visible and (c) infrared parts of the 59 and $80 \mathrm{~kW}$ spectra. The vertical dashed lines indicate the calculated IMFWM frequencies for a secondary pump at $625.2 \mathrm{~nm}$ propagating in the $\mathrm{LP}_{01}$ mode. Arrows indicate intra-modal FWM implying the main pump (see text).

spectral peaks in the visible between 400 and $500 \mathrm{~nm}$ [red line, Fig. 2(a)]. Also, a broad continuum of approximately 200-nm bandwidth started to develop around the pump [red line, Fig. 2(a)]. By increasing the power up to $80 \mathrm{~kW}$, we noted strong spectral broadening of all spectral components, as displayed by the black spectrum in Fig. 2(a). The characteristic asymmetric shape of the broadening of the first two sidebands (625 and $555 \mathrm{~nm}$ ) indicates that it is due to SRS [21]. Further details on the visible and infrared parts of the 59 and $80 \mathrm{~kW}$ spectra are shown in a frequency scale (shift from the primary pump) in Figs. 2(b) and 2(c).

To clarify the origin of the observed spectral components, we used the analytic formulation developed in Ref. [16], involving IMFWM between two pumps at the same wavelength $\lambda_{p}$ propagating in two distinct modes. The positions of the signal and idler waves can be found from the phase-matching and energy conservation conditions, given by

$$
\beta_{v}^{p}\left(\lambda_{p}\right)+\beta_{\kappa}^{p}\left(\lambda_{p}\right)-\beta_{\xi}^{s}\left(\lambda_{s}\right)-\beta_{\mu}^{i}\left(\lambda_{i}\right)=0 ; \quad \frac{2}{\lambda_{p}}=\frac{1}{\lambda_{s}}+\frac{1}{\lambda_{i}},
$$

where $\beta_{\mu}\left(\lambda_{j}\right)$ is the propagation constant of the $\mu$ th spatial mode at wavelength $\lambda_{j}$, and so on. The conversion efficiency can be defined by the coefficient $\sigma$ associated with the nonlinear coupling among the involved spatial modes:

$$
\sigma_{\mu v \kappa \xi}^{i p p s}=\iint \mathrm{d} x \mathrm{~d} y F_{\mu}^{*}\left(\lambda_{i}\right) F_{v}\left(\lambda_{p}\right) F_{\kappa}\left(\lambda_{p}\right) F_{\xi}^{*}\left(\lambda_{s}\right) .
$$

Here, $F_{\mu}\left(\lambda_{j}\right)$ is the spatial mode profile of the $\mu$ th mode at $\lambda_{j}$. The value of $\sigma$ is non-zero only for certain combinations of 


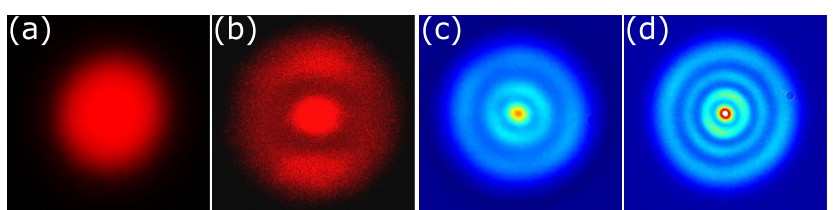

Fig. 3. Output beam profiles recorded with the camera at (a), (b) $625 \mathrm{~nm}$, (c) $473 \mathrm{~nm}$, and (d) $433 \mathrm{~nm}$ using a 10-nm bandpass filter.

modes, playing the role of a selection rule. The lack of information about the silica refractive index above $6 \mu \mathrm{m}$ prevents us from using the set of Eq. (1) to determine all the FWM sidebands. Hence, we considered the approximate analytic expression from Ref. [16]. The frequency shift $f_{k}$ of the FWM sidebands in a parabolic GRIN fiber can be approximated as

$$
f_{k}^{2} \approx \frac{c^{2} \sqrt{2 \Delta}}{\pi n^{\prime \prime}\left(\lambda_{p}\right)} \frac{k}{R \lambda_{p}^{3}},
$$

where $c$ is the speed of light in vacuum, and $n^{\prime \prime}$ is the second derivative of the fiber refractive index with respect to wavelength. $k$ is an integer giving the order of the considered sideband: it is related to the mode group numbers of the pump, signal, and idler spatial modes by $2\left(k+g_{p}\right)=g_{s}+g_{i}$, where the two pump photons are in the same spatial profile. In our configuration, we assumed that both pump photons belonged to the $\mathrm{LP}_{01}$ mode, so that $g_{p}=1$. Therefore, the selection rule $(\sigma \neq 0)$ imposes that the $k$ th-order sidebands can be composed of $\mathrm{LP}_{0 j}$ modes, where $j$ varies from 1 to $k+1$. In the particular case of the first order sidebands $(k=1)$, the Stokes line is generated in the $\mathrm{LP}_{01}\left(\right.$ or $\left.\mathrm{LP}_{02}\right)$ mode, while the anti-Stokes line is guided into the $\mathrm{LP}_{02}\left(\right.$ or $\mathrm{LP}_{01}$ ) mode. Let us mention that the parametric frequencies deduced from phase-matching conditions [Eq. (3)] are identical to those obtained from the collective approach $[11,12]$. However, the GPI sidebands in a highly MMF have a bell-shaped spatial distribution [12], which is completely different from the case of a FMF. Indeed, as can be seen from Figs. 3(a) and 3(b), by finely adjusting the injection conditions of light at the fiber input, the first-order antiStokes wave at $625.2 \mathrm{~nm}$ can propagate in either the $\mathrm{LP}_{01}$ or the $\mathrm{LP}_{02}$ mode. The associated values of $\sigma$ reported in Table 1 show that the most efficient frequency conversion should be the one involving an idler in the $\mathrm{LP}_{01}$ mode. This is in good agreement with our experiments, which show that the $625.2 \mathrm{~nm}$ wave is more intense when propagating in the $\mathrm{LP}_{01}$ mode (case of Fig. 2). In contrast, when the $625 \mathrm{~nm}$ wave propagated in the $\mathrm{LP}_{02}$ mode, its intensity was too low for the radiation to act as a secondary pump, and no line in the visible was generated. Thus the modal composition of radiation at $625 \mathrm{~nm}$ drastically affects the spectral extension at high power.

From Eq. (3), we found that the wavelengths associated with the first-order sidebands are 647.9 and $2982 \mathrm{~nm}$ $\left(f_{1}=181.2 \mathrm{THz}\right)$, as listed in Table 1 . The predicted idler

Table 1. Allowed First Order $(k=1)$ IMFWM Configurations for a $\mathrm{LP}_{01}$ Pump Profile ${ }^{a}$

\begin{tabular}{ccccccccc}
\hline $\boldsymbol{g}_{\boldsymbol{p}}$ & $\boldsymbol{g}_{\boldsymbol{i}}$ & $\boldsymbol{g}_{\boldsymbol{s}}$ & $\mathbf{P}$ & $\mathbf{I}$ & $\boldsymbol{S}$ & $\boldsymbol{\lambda}_{\boldsymbol{I}}(\mathbf{n m})$ & $\boldsymbol{\lambda}_{\boldsymbol{s}}(\mathbf{n m})$ & $\boldsymbol{\sigma}\left(\mathbf{1 0}^{-\mathbf{1 0}} \mathbf{m}^{-\mathbf{2}}\right)$ \\
\hline 1 & 1 & 3 & $\mathrm{LP}_{01}$ & $\mathrm{LP}_{01}$ & $\mathrm{LP}_{02}$ & 647.9 & 2982 & 1.04 \\
1 & 3 & 1 & $\mathrm{LP}_{01}$ & $\mathrm{LP}_{02}$ & $\mathrm{LP}_{01}$ & 647.9 & 2982 & 0.23 \\
\hline
\end{tabular}

${ }^{a} \mathrm{P}$, pump; I, idler; $\mathrm{S}$, signal. position is in agreement with our observations $(625.2 \mathrm{~nm})$, specifically if we consider the uncertainties on fiber parameters, which have been extrapolated at $1064 \mathrm{~nm}$ from the refractive index profile measured at $632 \mathrm{~nm}$ [Fig. 1(a)]. Note that for a pump at $1064 \mathrm{~nm}(282 \mathrm{THz})$, all anti-Stokes sidebands below $532 \mathrm{~nm}$ (see Fig. 2) - that is, beyond one octave from the pump-would have their Stokes counterparts at negative frequencies (their frequency shifts are higher than $282 \mathrm{THz}$ ). Hence, the process of conversion to these sidebands is unrealistic. The pump energy at $1064 \mathrm{~nm}$ is essentially transferred to the first anti-Stokes at $625.2 \mathrm{~nm}$, which then acts as a secondary pump: the generation of the peaks below $532 \mathrm{~nm}$ and the power saturation of the $625.2 \mathrm{~nm}$ sideband appear simultaneously when varying the input power [see Fig. 2(a)]. Moreover, the pulse duration of the parametric sidebands can be much shorter than the input pulse duration [22], leading to higher peak powers, thus significantly increasing the conversion efficiency of the corresponding cascaded IMFWM process. In our case, the pulse duration of the line at $625.2 \mathrm{~nm}$ was equal to $32 \mathrm{ps}$, when measured by using a fast photodetector (rise time of 14 ps) and a real-time, high-bandwidth oscilloscope $(50 \mathrm{GHz})$. The cascaded process can be seeded by spectral components in the vicinity of the main pump at $1064 \mathrm{~nm}$ (see Fig. 2 for the highest peak powers).

If we consider IMFWM induced by the $625.2 \mathrm{~nm}$ secondary pump in the $\mathrm{LP}_{01}$ mode, Eq. (3) implies that the generated sidebands are spaced by $\pm \sqrt{k} f_{1}$, with $f_{1}$ the detuning of the first order sideband and $k=1,2,3$, etc. The vertical dashed lines in Figs. 2(b) and 2(c) show their spectral positions, when calculated from Eq. (3). Table 2 compares some of the experimentally measured wavelengths with the calculated ones. As can be seen, the agreement is excellent for all seven listed sidebands. However, we observed only two Stokes waves, the first and sixth ones (with order -1 and -6 , respectively). The other Stokes waves of order between -2 and -4 are not visible certainly due to their low power compared to that of the wide spectral components on either side of the main pump. We explain the excellent agreement of Table 2 by the fact that the fiber parameters at $625 \mathrm{~nm}$ were determined with good accuracy, as they were derived from the index profile measured at a nearby wavelength, namely $632 \mathrm{~nm}$. By finely adjusting the injection conditions of light at the fiber input, it was also possible to observe individual higher-order spatial modes, as shown in Figs. 3(c) and 3(d) for the sidebands at 473 and $433 \mathrm{~nm}$ guided in the $\mathrm{LP}_{03}$ and $\mathrm{LP}_{04}$ modes, respectively. Moreover, the strong peak observed at $555.2 \mathrm{~nm}$ is the secondorder IMFWM sideband generated by the main pump at $1064 \mathrm{~nm}$. Whereas, less significant sidebands at $442 \mathrm{~nm}$ and around $865 \mathrm{~nm}$ [indicated by arrows in Figs. 2(b) and 2(c)] may be explained by intra-modal FWM processes. Indeed, the spectral bands around $865 \mathrm{~nm}$ [between S1 and S2 in Fig. 2(c)] are the mirror images (with respect to the main pump) of waves

Table 2. Experimental (Exp) versus Calculated (Calc) and Simulated (Sim) Parametric Wavelengths for a $\mathrm{LP}_{01}$ Secondary Pump

\begin{tabular}{lcccccccc}
\hline Order & $\mathbf{- 1}$ & $\mathbf{1}$ & $\mathbf{2}$ & $\mathbf{3}$ & $\mathbf{4}$ & $\mathbf{5}$ & $\mathbf{6}$ & \\
\hline Exp & 800.1 & 512.6 & 473.3 & 452 & 433.6 & 421.5 & 405.6 & $\mathrm{~nm}$ \\
Calc & 800.2 & 513.0 & 477.5 & 453.4 & 435.0 & 419.9 & 407.2 & $\mathrm{~nm}$ \\
Sim & 818 & 514 & 477 & 452 & 434 & 416 & - & $\mathrm{nm}$ \\
\hline
\end{tabular}




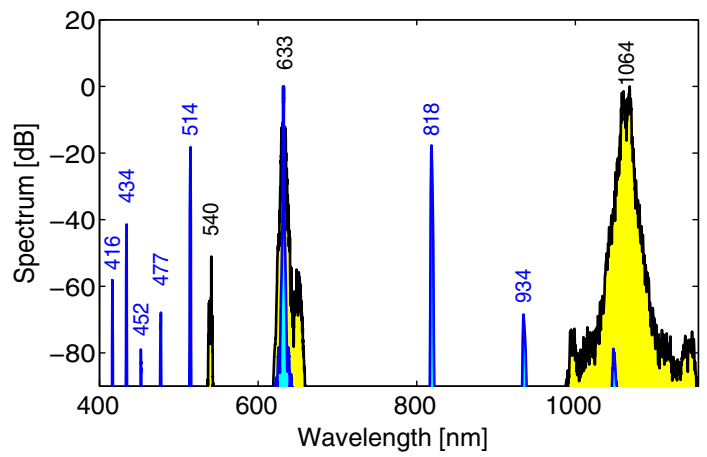

Fig. 4. Black curve: simulation for a 1064-nm pump, intensity $I=50 \mathrm{GW} / \mathrm{cm}^{2}$, fiber length $L=35 \mathrm{~cm}$. Blue curve: simulation for a $633-\mathrm{nm}$ pump, $I=20 \mathrm{GW} / \mathrm{cm}^{2}, L=10 \mathrm{~cm}$. The spectra are normalized to the corresponding level of the pump at 1064 and $633 \mathrm{~nm}$, respectively.

surrounding $1355 \mathrm{~nm}$ (S6). Whereas the component at $442 \mathrm{~nm}$ [between AS3 and AS4 in Fig. 2(b)] is the mirror image of the main pump with respect to the secondary pump.

To confirm our physical interpretations, we numerically solved the $(3+1) D$ nonlinear Schrödinger equation that reads as $[11,23]$

$$
\frac{\partial A}{\partial z}-i \frac{1}{2 k_{0}} \nabla_{\perp}^{2} A+i \frac{k^{\prime \prime}}{2} \frac{\partial^{2} A}{\partial t^{2}}+i \frac{k_{0} \Delta}{R^{2}} \rho^{2} A=i \gamma|A|^{2} A,
$$

with $A$ the complex field envelope expressed in a reference frame moving with the group velocity of the pump. $z$ is the propagation coordinate, and $\rho$ is the radial coordinate of the transverse domain. $k_{0}=\omega_{0} n_{o} / c$ and $k^{\prime \prime}$ is the group-velocity dispersion and $\gamma=\omega_{0} n_{2} / c$, being $n_{2}\left(3.210^{-20} \mathrm{~m}^{2} / \mathrm{W}\right)$, the nonlinear refractive index.

We directly solved Eq. (4) by using a standard split-step Fourier method. In the simulations, we fixed periodic boundary conditions in time $t$, while the transverse field is set to zero at the boundaries of the spatial window. In the simulations, we used an input Gaussian beam of $12 \mu \mathrm{m}$ FWHM diameter and a parabolic index profile fitting the experimental measurement [see Fig. 1(a)]. To reduce computational burden, the pump pulse duration was set to 9 ps. Raman scattering was not included, as it does not affect the FWM spectral positions. The black curve of Fig. 4 shows the simulated spectrum obtained for a pump at $1064 \mathrm{~nm}$. We can clearly see parametric sidebands due to IMFWM at $633 \mathrm{~nm}$ and at $540 \mathrm{~nm}$ (the corresponding Stokes sidebands in the infrared are not shown). These idler wavelengths are remarkably close to the experimental measurements $(625.2$ and $555.2 \mathrm{~nm}$ ) and to analytical values. To verify the role played by the secondary pump leading to cascaded IMFWM, we performed a separate numerical simulation with the pump wavelength set at $633 \mathrm{~nm}$. The results are shown by the blue curve of Fig. 4. There is a good agreement between numerical simulations of Eq. (4), analytic calculations, and experimental observations.

In conclusion, we reported the observation of far-detuned (from 200 up to $450 \mathrm{THz}$ ) frequency conversion via IMFWM in a multimode GRIN fiber supporting four modes at the pump wavelength. We identified a new cascaded nonlinear process. FWM generated directly from the main pump is quickly annihilated, since Stokes lines of an order higher than two cannot exist, their frequency shifts being larger than one octave. The first anti-Stokes sideband acts then as a secondary pump for the corresponding IMFWM processes. Such a cascaded process differs from what is generally observed for small detunings and harmonic sidebands, as sidebands exhibit anharmonically spaced frequency shifts. All nonlinear couplings were identified in our FMF experiments, without significant impact from SRS, in good agreement with phase-matching conditions and numerical simulations. From an application perspective, our observations open a new route for efficient frequency conversion and SC generation extended in the visible and near-UV.

Funding. iXcore Research Foundation; ANR Labex Action; Ministero dell'Istruzione, dell'Università e della Ricerca (MIUR) (PRIN 2015KEZNYM); Ministry of Education and Science of the Russian Federation (Minobrnauka) (14.Y26.31.0017); Horiba Medical and BPI France (dat@diag project).

Acknowledgments. We thank J. Fatome for the loan of the FMF.

${ }^{\dagger}$ These authors contributed equally to this Letter.

\section{REFERENCES}

1. R. Ryf, S. Randel, A. H. Gnauck, C. Bolle, A. Sierra, S. Mumtaz, M. Esmaeelpour, E. C. Burrows, R. Essiambre, P. J. Winzer, D. W. Peckham, A. H. McCurdy, and R. Lingle, J. Lightwave Technol. 30, 521 (2012).

2. A. Picozzi, G. Millot, and S. Wabnitz, Nat. Photonics 9, 289 (2015).

3. A. Mafi, J. Lightwave Technol. 30, 2803 (2012).

4. H. Pourbeyram and A. Mafi, Photonics 2, 906 (2015).

5. W. H. Renninger and F. W. Wise, Nat. Commun. 4, 1719 (2013).

6. L. G. Wright, D. N. Christodoulides, and F. W. Wise, Nat. Photonics 9 , 306 (2015).

7. L. G. Wright, W. H. Renninger, D. N. Christodoulides, and F. W. Wise, Opt. Express 23, 3492 (2015).

8. L. G. Wright, S. Wabnitz, D. N. Christodoulides, and F. W. Wise, Phys. Rev. Lett. 115, 223902 (2015).

9. K. O. Hill, D. C. Johnson, and B. S. Kawasaki, Appl. Opt. 20, 1075 (1981).

10. R. J. Essiambre, M. A. Mestre, and R. Ryf, IEEE Photon. Technol. Lett. 25, 539 (2013).

11. S. Longhi, Opt. Lett. 28, 2363 (2003).

12. K. Krupa, A. Tonello, A. Barthélémy, V. Couderc, B. M. Shalaby, A. Bendahmane, G. Millot, and S. Wabnitz, Phys. Rev. Lett. 116, 183901 (2016).

13. M. Droques, A. Kudlinski, G. Bouwmans, G. Martinelli, and A. Mussot, Opt. Lett. 37, 4832 (2012).

14. M. Conforti, S. Trillo, A. Mussot, and A. Kudlinski, Sci. Rep. 5, 9433 (2015).

15. N. J. Smith and N. J. Doran, Opt. Lett. 21, 570 (1996).

16. E. Nazemosadat, H. Pourbeyram, and A. Mafi, J. Opt. Soc. Am. B 33 , 144 (2016).

17. H. Pourbeyram and A. Mafi, Phys. Rev. A 94, 23815 (2016).

18. http://www.ofsoptics.com.

19. G. Millot, S. Pitois, P. Tchofo Dinda, and M. Haelterman, Opt. Lett. 22 1686 (1997)

20. M. Guasoni, Phys. Rev. A 92, 033849 (2015).

21. A. Mussot, T. Sylvestre, L. Provino, and H. Maillotte, Opt. Lett. 28, $1820(2003)$.

22. K. Krupa, C. Louot, V. Couderc, M. Fabert, R. Guenard, B. M. Shalaby, A. Tonello, A. Barthélémy, D. Pagnoux, P. Leproux, A. Bendahmane, R. Dupiol, G. Millot, and S. Wabnitz, Opt. Lett. 41, 5785 (2016).

23. S. S. Yu, C.-H. Chien, Y. Lai, and J. Wang, Opt. Commun. 119, 167 (1995). 